$\begin{aligned} & \text { Abstract PWE-111 Table } 2 \\
& \text { Action documented by clinician in } \\
& \text { response to a 'MUST' score of } \geq 1\end{aligned}$
\begin{tabular}{lll} 
MUST & Number of & Action taken \\
Score & Patients & \\
\hline Score 1 & 2 & Referred to the dietitian for advice \\
& 3 & No action documented \\
& 1 & Clinician mentioned weight loss in letter to the GP \\
& 1 & Clinician commented no further action needed \\
Score 2+ $2+$ & 1 & Cnown to the dietitian \\
& 1 & Clinician commented that score was inaccurate \\
& 1 & Referred to the dietitian \\
& 1 & Decision made by clinician for GP to monitor in \\
\end{tabular}

Conclusion The percentage of patients identified at risk of malnutrition ('MUST' score >1) was 25\%; in keeping with published UK data ${ }^{(3)}$. In $73 \%$ of patients an action was documented by the clinician in response to the screening result. This demonstrates that implementation of 'MUST' is feasible in a busy outpatient department, and that in the majority identified as high risk an appropriate action plan was implemented. We plan to cascade it across the remaining outpatient areas and improve compliance by further training and education.

1. NICE Clinical Guideline CG32: Nutrition support for adults: oral nutrition support, enteral tube feeding and parenteral nutrition (2006).

2. BAPEN http://www. bapen. org. uk/screening-and-must/mustcalculator

3. Russell CA, Elia M. Nutrition screening surveys in hospitals in England, 2007-2011: A report based on the amalgamated data from the four Nutrition Screening Week surveys undertaken by BAPEN in 2007, 2008, 2010 and 2011: BAPEN; 2014.

\section{PWE-112 ASSESSING KNOWLEDGE OF GLUTEN CONTAINING FOODS AMONGST HEALTHCARE PROFESSIONALS}

${ }^{1}$ Hasan Haboubi*, ${ }^{1}$ Adnan Ur Rahman, ${ }^{2}$ Aled Lloyd, ${ }^{1}$ Jill Swift. Department of Gastroenterology, Cardiff And Vale University Health Board, Cardiff, UK; ${ }^{2}$ Department of Medicine, AMBU Health Board, Swansea, UK

\subsection{6/gutjnl-2018-BSGAbstracts.346}

Introduction Coeliac disease affects approximately 1\% of the UK population and is a common reason for review in the Gastroenterology outpatient clinic. Furthermore, with growing evidence that gluten intolerance can lead to symptoms of IBS, there is an increasing demand on Gastroenterology services to be well equipped with knowledge about gluten containing products.

Methods We devised a structured questionnaire (scored out of 40) encompassing 4 main domains. These considered issues such as common foods which contained gluten, grains which contained gluten, foods which didn't contain gluten but may be contaminated with gluten during the manufacture process, and kitchen/food preparation which could lead to gluten contamination.

The survey was disseminated electronically to the WAGE database as well as the Cardiff and Vale University Health Board Medical mailing list.
Results There were a total of 115 complete responses (response rate of $10.5 \%$ ).

As expected individuals with known coeliac disease, or those living in a gluten free household scored highest (Median scores of $93 \%$ and $86.5 \%$ respectively) and were thus excluded from subsequent analysis.

Of the remaining 112 individuals, 47 (42\%) were doctors, 37 (33\% were nurses), 25 (22.3\%) were dietitians and the remainder were managers/admin staff.

Median scores showed dietitians scored best (85\%) followed by doctors (70\%), nurses (68\%) and managers $(55 \%)$, $\mathrm{p}<0.001$. GI dietitians scored better $(93 \%)$ than their non-GI counterparts, although this wasn't statistically significant, $\mathrm{p}=0.934$.

Within clinicians, paediatricians scored best (93\%), followed by surgeons $(n=3,73 \%)$, non-GI physicians $(n=17,67 \%)$, with Gastroenterologists ranked fourth $(n=17$, median score $=63 \%$ ). This was found to be dependent on grade, with Gastroenterology SpR's scoring worst amongst all clinical groups $(n=5$, median score $=38 \%), p=0.009$. Subgroup analysis demonstrated deficiencies in knowledge amongst Gastroenterology SpR's in domains such as awareness of gluten containing foods and foods which may be contaminated with gluten.

Conclusions Knowledge about gluten containing foods amongst clinicians is variable. Whilst it is reassuring that dietitians knowledge is best amongst those sampled, we acknowledge deficiency in knowledge amongst gastroenterologists compared to their non-GI counterparts, particularly amongst SpR's. Whilst the reasons underpinning this are not yet clear, further efforts to educate these clinicians who commonly manage coeliac patients may be required.

\section{PWE-113 NUTRITION SUPPORT TEAMS IN THE UK: THE CURRENT PICTURE}

${ }^{1}$ Thomas Hollingworth*, ${ }^{1}$ Tahir Akbar, ${ }^{2}$ Siddhartha Oke, ${ }^{3}$ Ruth McKee ${ }^{4}$ Andrew Rochford, ${ }^{5}$ Wendy-Ling Relph, ${ }^{6}$ Nikki Burch, ${ }^{1}$ Trevor Smith, ${ }^{2}$ Simon Gabe. ${ }^{1}$ University Hospital Southampton, Southampton, UK; ${ }^{2}$ St. Mark's Hospital, London, UK; ${ }^{3}$ Glasgow Royal Infirmary, Glasgow, UK; ${ }^{4}$ Newham University Hospital and Barts Health NHS Trust, London, UK; ${ }^{5}$ East Kent Hospitals University Foundation Trust, Kent, UK; ${ }^{6}$ University Hospitals Coventry and Warwickshire NHS Trust., West Midlands, UK

\subsection{6/gutjnl-2018-BSGAbstracts.347}

Introduction The importance of presence of a nutrition support team (NST) and a nutrition steering committee (NSC) were recognised in 1994 by BAPEN with the recommendation that all major UK hospitals should appoint a NSC and at least one NST. It was recognised that delivering excellent nutritional care within hospitals required the co-ordination and oversight of a NSC. The NST's role was to bring together a multi-disciplinary team that could support hospital staff in the provision of nutritional therapy, especially enteral and parenteral nutritional support, at a ward level. A NST should contain at least a doctor, nurse, dietician and pharmacist with specialist skills in nutritional support. NICE guidelines have also made recommendations about the provision of a NSC and NST in all acute trusts. In 2010 the NCEPOD report A Mixed bag, reported only $60.2 \%$ of hospitals in the UK as having a NST of which only $59 \%$ had a full MDT provision. We aimed to assess the current provision of NSC's and NST's within the UK at this present time. 
Methods In June 2017 BAPEN sent a freedom of information (FOI) request to all trusts in England, health boards in Scotland and Wales and social care trusts in Northern Ireland. This requested information on the size of the trust, the presence and composition of the NST and their role within the hospital along with information about the trust's NSC. Those responses received before 18 th October were collated. Hospitals with fewer than 100 beds were excluded from the analysis.

Results Of the 181 FOI requests made BAPEN received responses from 122 trusts representing 154 hospitals with more than 100 beds. $91 \%$ of trusts in the UK had a NSC and only $80 \%$ of trusts reported having a NST. Of this only $25 \%$ of social care trusts in Northern Ireland and $40 \%$ of health boards in Wales reported having an NST. 48\% of trusts with an NST had a nutrition nurse, a dietician, a doctor and a pharmacist. $76 \%$ of trusts with an NST had a nutrition nurse, $79 \%$ had a pharmacist, $86 \%$ had a doctor and $88 \%$ had a dietician. $57 \%$ of NSTs performed a ward round more than once a week with $10 \%$ seeing patients as required and $4 \%$ providing an advisory role only.

Conclusion There has been a clear improvement in the provision of NSC's and NST's within the UK over time. Sadly despite this increase in NSC's and NST's we have not managed to fulfil the aim of having one in each trust. Furthermore the majority do not have the full multi-disciplinary team provision required to provide the highest level of care. More work needs to be done to promote the importance of the NSC and NST and provide support in developing them in trusts that currently do not have them ensure that all trusts have access to them.

\section{PWE-114 GASTROSTOMY INSERTION: BEYOND THE MORTALITY RATES}

${ }^{1}$ Joanne Louise Hulley*, 'Satyasheel Ramful, ${ }^{1,2}$ Nick Thompson. ${ }^{1}$ Newcastle Hospitals, Newcastle, UK; ${ }^{2}$ Northern Nutrition Network, Newcastle, UK

\subsection{6/gutjpl-2018-BSGAbstracts.348}

Introduction Short-term mortality rates from percutaneous endoscopic gastrostomy (PEG) insertion are well documented and often the focus of audit. Our Northern Nutrition Network extends across nine trusts in the North East and Cumbria, comprising of gastroenterologists, dietitians and specialist nurses. We analysed our regional population.

Methods 3 months of prospective data were collected on patients undergoing PEG or radiologically inserted gastrostomy (RIG); focussing on mortality, premorbid state, complications and sedation use. 90 day outcomes were recorded.

Results 146 gastrostomies were inserted, with a 30 day mortality rate of $8.2 \%(12 / 146)$ and 90 day mortality rate of $17.5 \%$ (26/146). Our 30 day mortality included a $2 \%$ (3/146) risk of dying in the first 7 days after gastrostomy; deemed attributable to the procedure.

Indications for gastrostomy included; ENT/UGI obstruction/ malignancy (46/146), neurological conditions (20/146), stroke (31/146), depressed consciousness (17/146), malnutrition (4/146) and failure of function (13/146). 88 patients were female, 56 were male. The average ASA was 2.7; mean BMI was 23.5; mean albumin was 37; mean age was 66 years. The 30 day mortality group had a higher mean age (76.7 vs. 66 years) and ASA (3.1 vs. 2.7$)$, and a lower BMI (18.5 vs. 23.5$)$ and albumin (31 vs. 37), suggesting these factors, which are associated with frailty, could impact on mortality.

We found a complication rate of $27 \%(40 / 146)$ and a statistically significant link between mortality and complications. The 30 day mortality increased from $4.7 \%$ (5/106) to $17.5 \%$ $(7 / 40)$ if a complication occurred $(p=0.012)$. This was reflected in the 90 day mortality group, where mortality increased from $13 \%(14 / 106)$ in the group with no complications to $30 \%(12 / 40)$ in the complication group $(p=0.018)$.

The most common complication was pneumonia, at $11 \%$ (16/147). The risk of pneumonia could be linked with sedation use, as the group receiving midazolam sedation (average $2 \mathrm{mg}$ per patient) had a pneumonia rate of $13.7 \%(15 / 109)$ whereas in the un-sedated group only one patient suffered pneumonic complications $(n=11)$. There were no reported cases of pneumonia in the 26 patients who underwent general anaesthetic for the procedure.

Conclusion We report similar mortality rates to previous studies. Our data follow the trend that older, sicker, less wellnourished patients have poorer outcomes following gastrostomy insertion than their younger, fitter, counterparts. We have shown a statistically significant link between increasing mortality and complications. Likewise, our data suggest that sedation is a risk factor for our most common complication; pneumonia. Careful patient selection and realistic conversations with patients and relatives prior to gastrostomy insertion remain paramount.

\section{PWE-115 DIETARY PATTERNS IN INFLAMMATORY BOWEL DISEASE-INTOLERANCES, QUALITY OF LIFE AND CALCIUM/VITAMIN D INTAKE}

${ }^{1}$ Rajesh Krishnamoorthy*, ${ }^{2}$ Yvonne Jeanes. ${ }^{1}$ Derby Teaching Hospitals NHS Foundation Trust, Derby, UK; ${ }^{2}$ University of Roehampton, London, UK

\subsection{6/gutjinl-2018-BSGAbstracts.349}

IntroductionFood intolerances and food avoidance are common in inflammatory bowel disease (IBD). This cross-sectional study explored the prevalence of food intolerance patterns in IBD and assessed the food related quality of life (FR-QoL) and vitamin $\mathrm{D}$ and calcium intake in IBD patients.

Methods An online questionnaire with detailed questions relating to food groups commonly avoided, food related quality of life tool and calcium and vitamin D intake was displayed on the Crohn's and Colitis UK website. Disease activity was assessed by the Minnesota IBD activity index, which is a validated, patient-defined tool that relates to the patient's perception of IBD activity over the last six months. FR-QoL was assessed by a validated questionnaire (FR-QoL-29) which comprises of 29 statements encompassing different psychosocial aspects surrounding food and eating from an IBD symptoms perspective. Details of the type of IBD, duration of disease, previous surgery and disease activity were collected. Fishers' exact test and Pearson correlation were used for statistical analysis.

Results 67 respondents (40 Crohn's, 23 ulcerative colitis, 2 unclassified and 2 microscopic colitis) participated in the survey. Food avoidance was seen in 65 (97\%) patients, with mean number of foods avoided at 6 . Vegetables were avoided in $60 \%$ of the patients, followed by wheat-based products in $56 \%$ of patients. $82 \%$ of patients reported that their IBD was active. Food related quality of life was poor in inflammatory bowel disease patients and disease activity significantly 